\title{
Response of Grained $\mathrm{La}_{0.67} \mathrm{Ca}_{0.33} \mathrm{MnO}_{3}$ Films to Microwave Radiation and the Method for Measuring Electrical Resistance of Grains
}

\author{
K. Repšas, A. LaurinaviČIUs, R.-A. VaŠKeviČIus, \\ F. Anisimovas, A. Deksnys and B. Vengalis
}

Semiconductor Physics Institute, Goštauto 11, 01108 Vilnius, Lithuania

(Received June 26, 2006)

\begin{abstract}
It was found that the response of grained $\mathrm{La}_{0.67} \mathrm{Ca}_{0.33} \mathrm{MnO}_{3}$ films to microwave radiation is of thermal nature. A nonresonant method for measuring of the electric resistance of grains was developed. It allows one to avoid the influence of the magnetic field of the wave on the measurement results. The measurements of the temperature dependences of the grain electric resistance indicate that in the vicinity of the maximum-resistance temperature $T_{\mathrm{m}}$, the intrinsic electric resistance of the grains is more than by two orders of magnitude lower than the film resistance measured by applying the dc current. The obtained experimental results agree well with those given by resonant techniques.
\end{abstract}

PACS numbers: 75.47.Lx, 75.47.De

\section{Introduction}

During recent years, much attention has been paid to manganites possessing perovskite structure, whose general formula reads $\mathrm{R}_{1-x} \mathrm{M}_{x} \mathrm{MnO}_{3}$ (here $\mathrm{R}=\mathrm{La}$, $\mathrm{Nd}, \mathrm{Pr} ; \mathrm{M}=\mathrm{Ca}, \mathrm{Sr}, \mathrm{Ba}$, and $\mathrm{Pb}$ ). At $x=0.2-0.4$, many of these compounds show very large (giant) magnetoresistance. Just for this reason, they are very important in the fabrication of various electronic equipment. By using thin films of these substances, novel electric devices are expected to be created: integral elements of magnetic memory, information recording and reading heads, various magnetic field sensors, etc. Lattice parameters of these compounds are close to many other perovskites (ferroelectrics and high-temperature superconductors), therefore, by no doubt, new application possibilities will be opened once manganite heterostructures with other metal oxides will be fabricated [1-5]. 
Using dc and microwave current, single crystals of some magnetics $[6,7]$ were studied, as well as their thin films [8-11]. Laser-generated light pulses were also employed for these investigations [12-14].

The resonant technique of measurements is most widely employed. Almost all known effects are explored by this method, including electric resistance of grains and magnetic impedance [6, 9-11], ferromagnetic resonance and antiresonance $[2,9]$, absorption of waves by a film in the presence of magnetic field and without it [15-21], resonant response of manganite to either a low-frequency ac electric field has been induced in the film along with the microwave electric field [22], or a dc electric field has been induced along with the electric field generated by a laser light pulse [13], and some other effects.

In the paper [17], the authors compared the absorbed microwave power $P_{\mathrm{ab}}$ measured in the film by the resonant technique with the values obtained by employing the conventional method (i.e. by measuring the incident $P_{\mathrm{i}}$, reflected $P_{\mathrm{r}}$, and transmitted $P_{\mathrm{t}}$ microwave powers). The results of both methods coincided well.

One of the most important questions in the understanding of the mechanism of the giant magnetoresistance is the following: what role in its formation is played by the granules and what by the intergranular contacts? This question could be answered in part by comparing the total dc resistance of the film to the real part of the complex resistance of the granules.

In this paper we present a nonresonant method for measuring of the electric resistance of grains $R_{\mathrm{gr}}(T)$. Its essence is that the intrinsic electric conductivity of the grains $\sigma_{\mathrm{gr}}(T)$ is obtained from the average power $P_{\mathrm{ab}}$ absorbed by the film during one period, which can be expressed as

$$
P_{\mathrm{ab}}=\frac{1}{2} \sigma_{\mathrm{gr}}(T) E_{\mathrm{mp}}^{2} V_{\mathrm{p}} \cos \varphi .
$$

Here $E_{\mathrm{mp}}$ is the ac electric field strength in the film, $V_{\mathrm{p}}$ the film volume, and $\cos \varphi$ the cosine of the phase angle between the ac current density and ac electric field strength in the film, and $\varphi=\varphi_{\varepsilon}+\varphi_{\mu}$ with $\varphi_{\varepsilon}\left(\varphi_{\mu}\right)$ being the phase angle due to the dielectric (magnetic) permittivity $\varepsilon \varepsilon_{0}\left(\mu \mu_{0}\right)$ of the film.

When the chosen sample dimensions distort only slightly the electromagnetic field of the travelling wave in the waveguide, one can expect that the field in the film is the same as in the empty waveguide at the same point of sample location. Then the electric field strength in the film may be obtained from the general relationship between the travelling wave electric field strength in the waveguide and its power. The estimated contribution of the film permittivity $\varepsilon \varepsilon_{0}$ into the angle $\varphi$ revealed that $\varphi_{\varepsilon} \approx 0$.

The paper [1] presents the results on magnetic permeability $\mu^{*}=\mu^{\prime}+\mathrm{i} \mu^{\prime \prime}$, which indicate that in thin polycrystalline $\mathrm{La}_{1-x} \mathrm{Ca}_{x} \mathrm{MnO}_{3}$ films, with $x=0.3$, we have $\mu^{\prime} / \mu_{0} \approx-1.1$ and $\mu^{\prime \prime} / \mu_{0} \approx 2$ in the ferromagnetic state, and $\mu^{\prime} / \mu_{0} \approx 1$ and $\mu^{\prime \prime} / \mu_{0} \approx 0$ at room temperature. Such large permeability values in 
the ferromagnetic state must give large contributions to $\cos \varphi$. This contribution can be avoided by measuring the power $P_{\mathrm{ab}}$ absorbed by the film under the standing wave mode, when the sample is at the electric field maximum of the wave, where its magnetic field is zero.

The main aims of this paper were as follows:

i) To establish the nature of the response to microwave radiation of films.

ii) To find temperature dependence of the grain electric resistance.

iii) To compare the results with the literature data.

\section{Investigation of the film response to microwave radiation}

After solving the thermal conductivity equation for the case when dc current density $j_{0}$ and microwave current density of amplitude $j_{\mathrm{m}}\left(j_{\mathrm{m}} \gg j_{0}\right)$ flow along a rectangular manganite sample of length $l$, width $b$, and thickness $d$, with one surface of the film adiabatically isolated (temperature $T(0)$ ) and the other surface thermostated (constant temperature $T(d)$ ), the following temperature difference is obtained between the two surfaces of the film:

$$
T(0)-T(d)=\frac{\sigma}{4 \kappa} E^{2} d^{2} .
$$

Here $\kappa$ is the intrinsic thermal conductivity, $\sigma$ the intrinsic electrical conductivity, and $E=E_{0}+\tilde{E}$ the total electric field strength with $E_{0}$ and $\tilde{E}$ being the strength of the dc field and the wave, respectively. It can be concluded from Eq. (2) that the thermal response should have the following properties:

i) The response must be determined by the increase in the film resistance when its temperature $T$ is lower than $T_{\mathrm{m}}$ at which one observes the maximum in the dependence of the resistance on the temperature $T$.

ii) If $T(d)$ is kept constant, then the temperature difference or thermal response should depend linearly on the square of the wave electric field amplitude, or on the radiation power.

iii) At constant $T(d)$ and the same radiation power, the response is larger when the film is thicker.

The experiment was carried out with two $\mathrm{La}_{1-x} \mathrm{Ca}_{x} \mathrm{MnO}_{3}$ films of different thickness $(0.15$ and $1.30 \mu \mathrm{m})$ fabricated on crystalline $\operatorname{MgO}(100)$ substrates by laser evaporation from the ceramic target of the same chemical composition. The width of the samples was $3 \mathrm{~mm}$, and the distance between ohmic contacts attained $1 \mathrm{~cm}$.

Microwave measurements were performed at the liquid nitrogen temperature. A simplified scheme of the measurements is shown in Fig. 1. The setup consists of a $23 \times 5 \mathrm{~mm}^{2}$ waveguide head $B$, through which the sample $M$ is inserted and placed at the electric field maximum. The dc circuit is composed of the voltage supply $U_{0}$, sample $R_{\mathrm{p}}$, and ohmic resistance $R$ connected in series; the latter is much smaller than the sample resistance $R_{\mathrm{p}}$. 


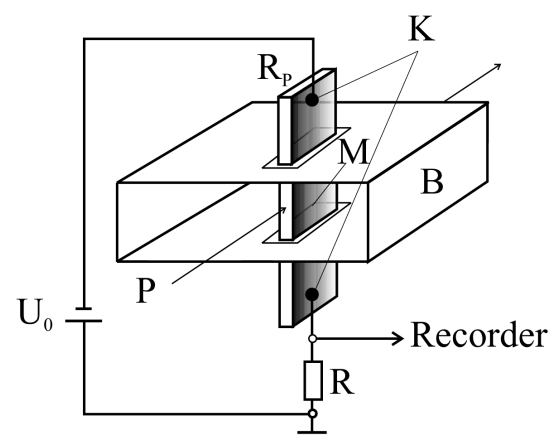

Fig. 1. The scheme of microwave measurement setup. $B$ is the waveguide head, $R_{\mathrm{p}}-$ the resistance of the film, $M$ - the film under study, $R$ - the ohmic resistance, $K-$ the contacts, $U_{0}$ is the dc voltage supply, and $P$ is the microwave radiation power.

Due to the action of the meander-modulated microwave radiation $(f=$ $10 \mathrm{GHz}$ ) on the film, the direct current change, and the dependence of the response upon the radiation power is plotted by a recorder.

Power-voltage characteristics of the samples are presented in Fig. 2. As seen, they are linear for both samples at larger radiation powers, but when continued from the higher-power region to the lower-power one, the characteristics intersect the power axis at different points (shown by arrows in the plot). For the thicker film (1), the intersection is closer to the coordinates origin than for the thinner one (2). This is because no response arises if the amount of heat generated by the radiation is small and can be drained to the substrate. In the thicker film, the heat removal is less efficient, because nonequilibrium phonons generated by radiation must pass a longer way.

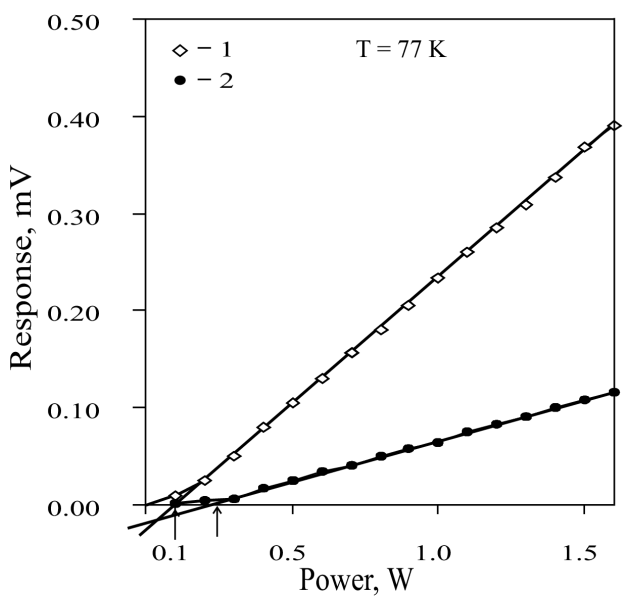

Fig. 2. Power-voltage characteristics of the thick (1) and thin (2) film. 
Thus, we see that the above-mentioned properties of the thermal response are clearly seen in the experiment. Therefore, we can conclude that the observed response has a thermal nature.

On this basis, we propose the following model of the film response to microwave radiation.

Electric capacitance of intergrain boundaries by-passes their ohmic resistance, therefore, microwave radiation heats only grains, which in their turn heat up the intergrain boundaries and so change their ohmic resistance. Since the main contribution to the film resistance is brought by the intergrain resistance, its variation changes efficiently the dc current that flows across the sample and thus changes the voltage drop across the resistance connected in series to the sample, thus generates the sample response to the microwave radiation.

\section{Microwave study of electric resistance of grains}

To eliminate $\cos \varphi_{\mu}$ and to valuate the grain resistance $R_{\mathrm{gr}}(T)$ we measure $P_{\mathrm{ab}}\left(T_{0}\right)$ under room temperature $T_{0}$, where $\cos \varphi=1$ in the travelling wave mode $P_{\mathrm{ab} 1}\left(T_{0}\right)$, and then in standing wave electric field maximum $P_{\mathrm{ab} 2}\left(T_{0}\right)$. By using the same response, we reach the equality $P_{\mathrm{ab} 1}=P_{\mathrm{ab} 2}$. Then we have

$$
P_{\mathrm{ab} 2}\left(T_{0}\right)=\frac{1}{2} \sigma_{\mathrm{gr}}\left(T_{0}\right) E_{\mathrm{m}}^{2}\left(T_{0}\right) V_{\mathrm{p}}=P_{\mathrm{ab} 1}\left(T_{0}\right)
$$

Maintaining the same values of incident $P_{\mathrm{i}}$ microwave power but varying the temperature $T$, we get in the standing wave mode

$$
P_{\mathrm{ab} 2}(T)=\frac{1}{2} \sigma_{\mathrm{gr}}(T) E_{\mathrm{m}}^{2}(T) V_{\mathrm{p}}
$$

Since, as mentioned before, the distortion of the field in the waveguide due to the presence of the sample is small, and the power of the incident wave is kept constant $\left(P_{\mathrm{ab} 2}(T) \approx\right.$ const $)$, one may expect that $E_{\mathrm{m}}^{2}\left(T_{0}\right) \approx E_{\mathrm{m}}^{2}(T)$. Then from $(3)$ and $(4)$ we obtain

$$
R_{\mathrm{gr}}(T)=R_{\mathrm{gr}}\left(T_{0}\right) \frac{P_{\mathrm{ab} 2}\left(T_{0}\right)}{P_{\mathrm{ab} 2}(T)}
$$

Thus, using (3) to express $R_{\mathrm{gr}}\left(T_{0}\right)$ and measuring the ratio $\left(P_{\mathrm{ab} 2}\left(T_{0}\right) / P_{\mathrm{ab} 2}(T)\right)$ we are able to determine the dependence of the grain resistance on the temperature $T$ :

$$
R_{\mathrm{gr}}\left(T_{0}\right)=\frac{1}{\sigma_{\mathrm{gr}}\left(T_{0}\right)} \frac{l_{\mathrm{p}}}{S_{\mathrm{p}}}=\frac{1}{2 P_{\mathrm{ab} 1}\left(T_{0}\right)} E_{\mathrm{m}}^{2}\left(T_{0}\right) l_{\mathrm{p}}^{2}
$$

Here, $l_{\mathrm{p}}$ and $S_{\mathrm{p}}$ denote the length and cross-section of the film under study.

From Eqs. (3) and (5), by making use of the relationship $E_{\mathrm{m}}^{2}\left(T_{0}\right)=\gamma^{2} P_{\mathrm{i} 1}\left(T_{0}\right)$ [23], we obtain that

$$
R_{\mathrm{gr}}\left(T_{0}\right)=\frac{1}{2} \gamma^{2} l_{\mathrm{p}}^{2} \frac{P_{\mathrm{i} 1}\left(T_{0}\right)}{P_{\mathrm{ab} 1}\left(T_{0}\right)} .
$$

Experiments were performed on polycrystalline $\mathrm{La}_{1-x} \mathrm{Ca}_{x} \mathrm{MnO}_{3}$ films, which were prepared by thermal evaporation of $\mathrm{LaF}_{2}, \mathrm{CaF}_{2}$, and $\mathrm{Mn}$ on $\mathrm{MgO}$ substrates, 
with subsequent thermal removal of $\mathrm{F}_{2}$ and final crystallization of the compound. The sample dimensions were $1 \times 0.4 \times 2 \times 10^{-5} \mathrm{~cm}^{3}$.

Microwave measurements were performed by using the setup depicted in Fig. 1. Travelling and standing waves were obtained by switching the waveguide channel between the matched load and the short-circuit piston. A power meter with the accuracy of $10 \%$ was employed to measure the radiation power incident on the sample, while the radiation power reflected from the sample was evaluated by using couplers and detectors. The value of the coefficient was estimated by the formula taken from the handbook [23], taking into account that we measure the power average over one period

$$
P=\frac{a l_{\mathrm{b}}}{4 Z_{\mathrm{b}}} E_{1}^{2}
$$

Here $a, l_{\mathrm{b}}$, and $Z_{\mathrm{b}}$ are the width of the waveguide wide wall, its height, and wave resistance, respectively, and $E_{1}$ is the electric field strength at the centre of the waveguide wide wall. In our case, $a=2.3 \mathrm{~cm}, l_{\mathrm{b}}=0.5 \mathrm{~cm}$, and $Z_{\mathrm{b}}=497.3 \Omega$, therefore, $\gamma^{2}=1730 \Omega / \mathrm{cm}^{2}$.

The measured temperature dependence of the grain resistance $R_{\mathrm{gr}}(T)$ is shown in Fig. 3. For comparison, the temperature dependence of the film resistance $R_{\mathrm{p}}(T)$ measured by dc current is shown. As follows from Fig. 3, the temperature dependence of the grain resistance $R_{\text {gr }}(T)$ has a broadened maximum which is shifted towards higher temperatures with respect to film dc resistance $R_{\mathrm{p}}(T)$ maximum. Moreover, in the vicinity of the $R_{\mathrm{p}}(T)$ maximum temperature, the grain resistance $R_{\mathrm{gr}}(T)$ is lower by more than two orders of magnitude than the film resistance $R_{\mathrm{p}}(T)$. This is in good agreement with the results reported in $[10,11]$.

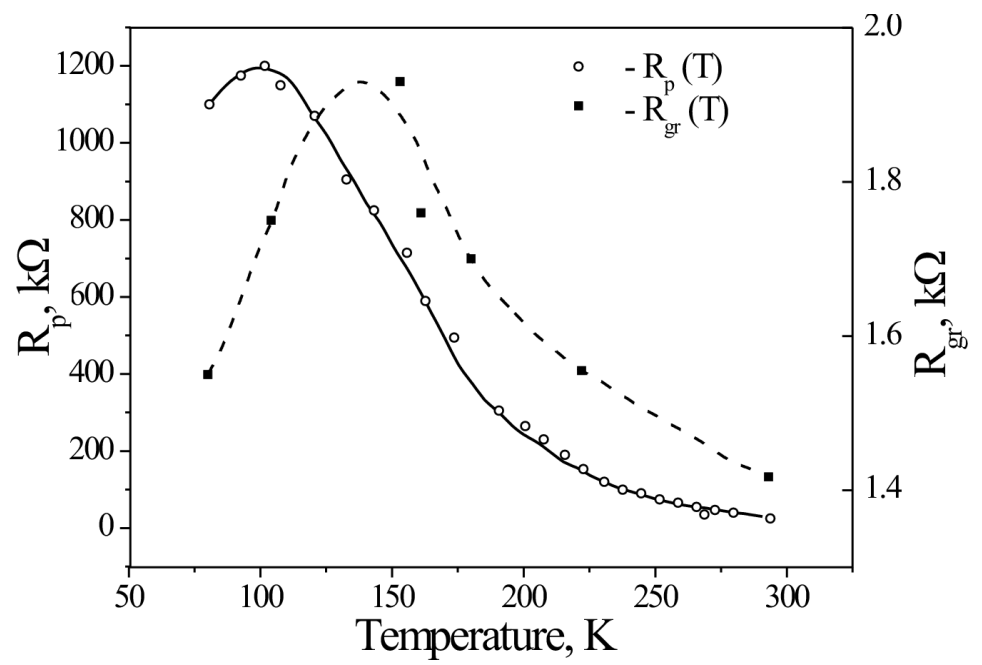

Fig. 3. The dependences of the sample resistance $R_{\mathrm{p}}(T)$ and grain resistance $R_{\mathrm{gr}}(T)$ on temperature $T$. 


\section{Conclusions}

1. It has been found that in our case, the film response to microwave radiation is of thermal nature.

2. A model of the film response to microwave radiation is proposed. According to this model, due to the shunting of the intergrain ohmic resistances by the electric capacitance, the microwave radiation mostly heats the granules which, in their own turn, heat the intergrain boundaries thereby modifying their ohmic resistance and the magnitude of the dc current flowing in the film.

3. The developed nonresonant method for measuring of the electrical resistance of the grains allows one to avoid the influence of the phase angle $\varphi$ between the ac current density and the ac electric field strength, which is caused by magnetic permeability $\mu$, on the measurement results. This is achieved by finding the intrinsic electric resistance of the grains from the value of the radiation power absorbed in the film placed at the electric field maximum point in the standing wave mode, when the magnetic field of the wave is zero.

4. The efficiency of the method is proved by the fact that temperature dependences of the grain electric resistance measured by the proposed method coincide well with the literature data obtained by resonant techniques.

5. The fact that the resistance of the granules is three orders of magnitude lower than the resistance of the film proves that the crucial role in the formation of the giant magnetoresistance is played by the magnetic field induced change of the resistance of the intergranular contacts.

\section{References}

[1] B. Vengalis, A.G. Oginskis, V. Lisauskas, N. Shiktorov, V. Jasutis, S.A. Karpinskas, A. Česnys, A. Maneikis, Mater. Sci. Forum. 297-298, 303 (1999).

[2] S.E. Lofland, M. Dominguez, S.D. Tyagi, S.M. Bhagat, M.C. Robson, T. Venkatesan, R. Ramesh, I. Takeuchi, Z. Trajanovic, C. Kwon, Thin Solid Films 288, 256 (1996).

[3] C.M. Hu, J. Nitta, A. Jensen, J.B. Hansen, H. Takayanagi, T. Matsuyama, D. Heitmann, U. Merkt, J. Appl. Phys. 91, 7251 (2002).

[4] T. Manago, H. Akinaga, Appl. Phys. Lett. 81, 694 (2002).

[5] J.B. Philipp, J. Klein, C. Recher, T. Walther, W. Mader, M. Schmid, R. Suryanarayanan, L. Alff, R. Gross, Phys. Rev. B 65, 184411 (2002).

[6] S.E. Lofland, S.M. Bhagat, S.D. Tyagi, Y.M. Mukowskii, S.G. Karabashev, A.M. Balbashov, J. Appl. Phys. 80, 3592 (1996).

[7] A. Pimenov, M. Biberachev, D. Ivannikov, A. Loidl, V.Yu. Ivanov, A.A. Mikhin, A.M. Balbashov, Phys. Rev. B 62, 5685 (2000). 
[8] S.I. Patil, S.M. Bhagat, Q.Q. Shu, S.E. Lofland, S.B. Ogale, V.N. Smolianinova, X. Zhang, B.S. Palmer, R.S. Decca, F.A. Brown, H.D. Drew, R.L. Greene, J.O. Troyanchuk, W.M. Mc Carrol, Phys. Rev. B 62, 9548 (2000).

[9] N.J. Solin, A.A. Samokhvalov, S.V. Naumov, Phys. Solid State 40, 1706 (1998).

[10] N.J. Solin, S.V. Naumov, A.A. Samokhvalov, Phys. Solid State 42, 925 (2000).

[11] K.A. Yates, L.F. Cohen, C. Watine, T.-N. Tay, F. Damay, J. MacManus-Drisol, R.S. Freitas, L. Ghivelder, E.M. Haines, G.A. Gehring, J. Appl. Phys. 88, 4703 (2000).

[12] M.-T. Hong, Y.-C. Chen, C.-C. Hsu, W.-C. Wu, T.-C. Chow, H. Chow, Jpn. J. Appl. Phys. 40, 4886 (2001).

[13] H.Y. Hwang, S.-W. Cheong, B. Batlogg, Appl. Phys. Lett. 68, 3494 (1996).

[14] A. Gilabert, A. Plecenik, K. Fröhlich, Š. Gaži, M. Pripko, Ž. Mozolova, D. Machajdik, Š. Benacka, M.G. Medici, M. Grajcar, P. Kuš, Appl. Phys. Lett. 78, 1712 (2001).

[15] S.D. Tyagi, S.E. Lofland, M. Dominguez, S.M. Bhagat, C. Kwon, M.C. Robson, R. Ramesh, T. Venkatesan, Appl. Phys. Lett. 68, 2893 (1996).

[16] F.J. Owens, J. Appl. Phys. 82, 3054 (1997).

[17] V.V. Srinivasu, S.E. Lofland, S.M. Bhagat, K. Ghosh, S.D. Tyagi, J. Appl. Phys. 86, 1067 (1999).

[18] Q.Q. Shu, S.M. Bhagat, S.E. Lofland, I.O. Troyanchuk, Solid State Commun. 109, 73 (1998).

[19] A. Rinkevich, A. Nossov, V. Vassiliev, V. Ustinov, Phys. Status Solidi A 179, 221 (2000).

[20] G. Li, G.-G. Hu, H.D. Zhou, X.J. Fan, X.-G. Li, J. Appl. Phys. 90, 5512 (2001).

[21] D.L. Lyfar, S.M. Ryabchenko, V.N. Krivoruchko, S.I. Khartsev, A.M. Grishin, Phys. Rev. B 69, 100409(R) (2004).

[22] N.V. Volkov, G.A. Petrakowskii, K.A. Sablina, S.V. Koval, Phys. Solid State 41, 1842 (2001).

[23] Waveguide Handbook, Ed. N. Marcuvitz, McGraw-Hill, New York 1986, p. 62. 